\title{
Incidence and management of post-dural punction headache after epidural for labour. A review of 2703 obstetric epidurals in a single centre during two years.
}

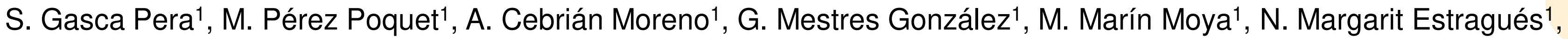
N. Párraga Fernández², J. Balsells Feltrer ${ }^{1}$.

${ }^{1}$ Hospital Universitari Mutua Terrassa, Anesthesiology, Terrassa, Spain.

${ }^{2}$ Hospital Universitari Mutua Terrassa, Acute Pain Unit Nurse, Terrassa, Spain.

\section{Background and Aims}

Post-dural puncture headache (PDPH) is the most common complication following accidental dural puncture (ADP) and is particularly frequent after epidural analgesia in obstetrics. The aim of our study is to describe the incidence and management of PDPH after epidural analgesia in obstetric patients in our hospital, as well as the efficacy of our PDPH protocol in clinical practice.

\section{Methods}

This is a retrospective observational study. We included all obstetric patients $(n=2703)$ who received epidural anaesthesia for labour during 2016 and 2017. Patients with reported ADP and those in whom ADP was not noticed but developed PDPH, started postpartum standardized treatment for PDPH. It included conservative therapies such as bed rest, hydration, corticoids and analgesics. If headache continued after 48 hours, an epidural blood patch (EBP) was indicated. Incidence of PDPH, patients who received EBP, the day in which EBP was made and the remission of headache were recorded.

\section{Results}

The incidence of reported obstetric ADP in our centre was $1.15 \%$ (31/2703), $64.5 \%$ (20/31) of cases developed PDPH. EBP was performed in $65 \%$ (13/20) of women with persistent headache and was resolved in 12/13 (92.3\%). One case needed a second EBP. The median until the EBP was performed was 4 days (range 3-6).

\section{Conclusions}

The incidence of PDPH after ADP in our centre is within the published average (0.5-2.5\%). The EBP is a useful technique for treatment in patients with unsuccessful conservative management of PDPH.

\section{Patients recieving epidural anesthesia for labour 2016-2017}

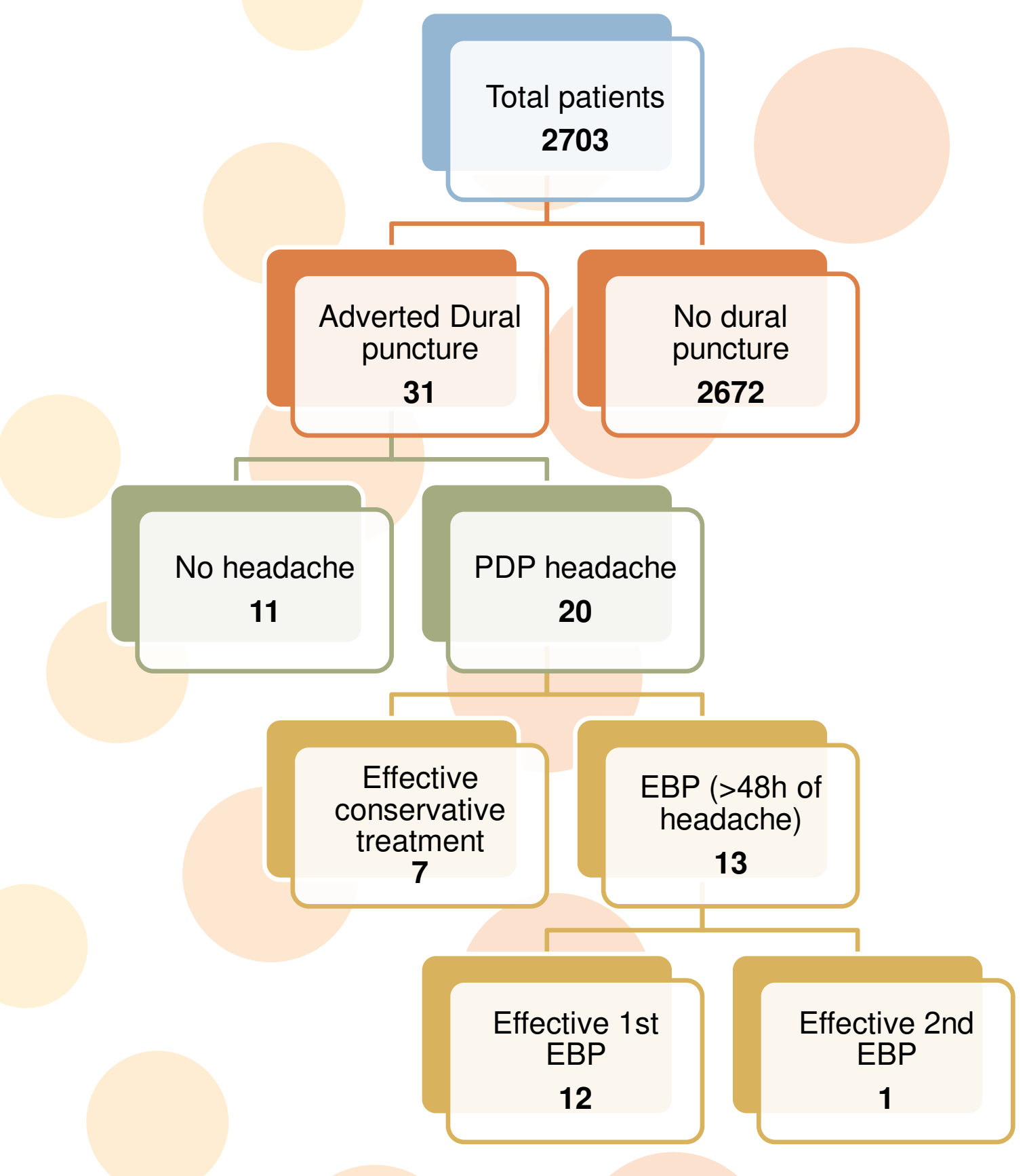

Figure 1. Number of patients recieving epidural anesthesia for labour during 2016-2017 in our hospital.

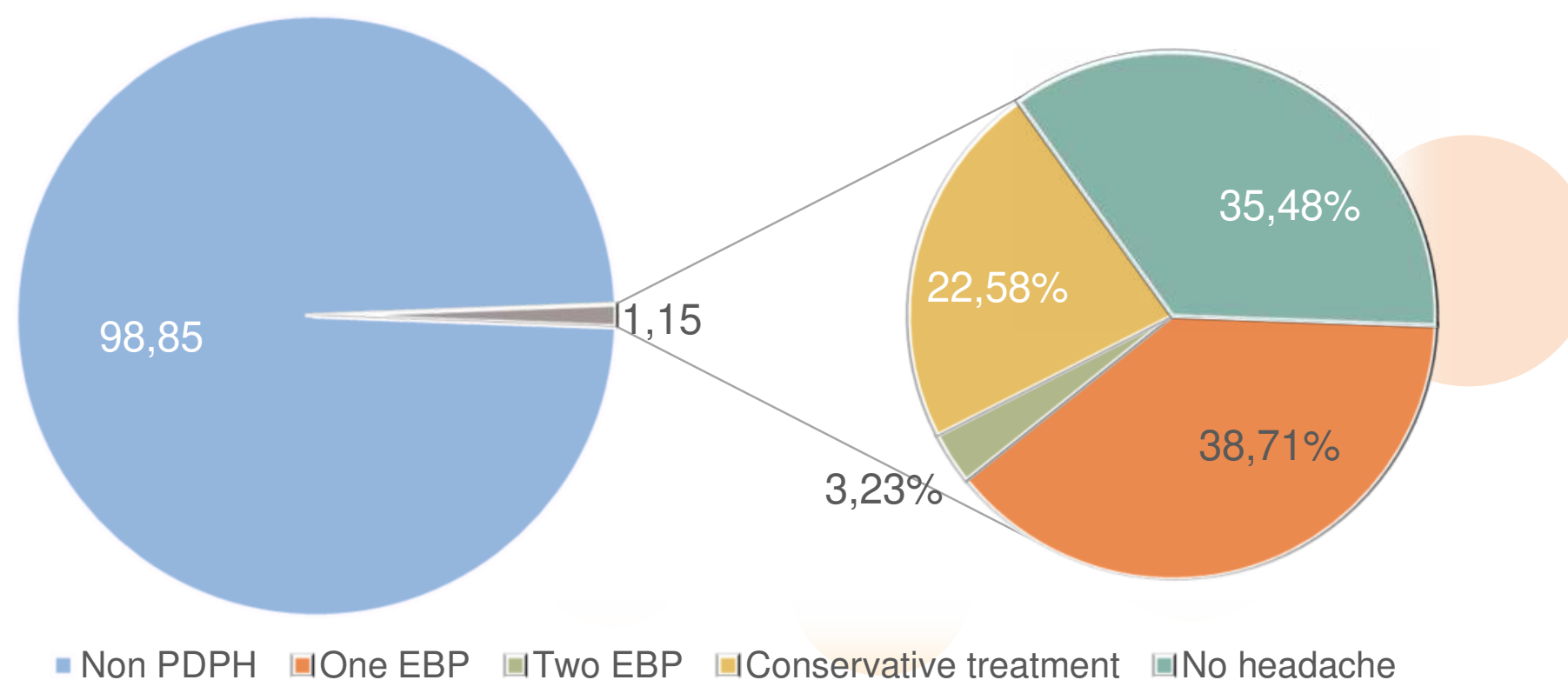

Figure 2. Incidence of patients with ADP in our centre during 2016-27 and percentage in each treatment group.

1. Gaisier R. R. Postdural puncture headache: an evidence-based approach. Anesthesiol Clin. 2017 Mar;35(1):157-167

2. Booth J. L., Pan P. H., Thomas J. A., Harris L. C., D'Angelo R. A retrospective review of an epidural blood patch database: the incidence of epidural blood patch associated with obstetric neuraxial anesthetic techniques and the effect of blood volume on efficacy. Int J Obstet Anesth. 2017 Feb;29:10-17 3. Suescun H, Austin P, Gabaldon D. Nonpharmacologic Nauraxial Interventions for Prophylaxis of Postdural Puncture Headache in the Obstetric Patient. AANA J 2016. Feb 84(1): 15-22

4. López T., Garzón J. C., Sánchez F. J., Muriel C. Cefalea postpunción duran en obstetricia. Rev Esp Anestesiol Reanim. 2011;58:563-573 